Article

\title{
Track Allocation Optimization in Multi-Direction High-Speed Railway Stations
}

\author{
Qin Zhang, Xiaoning Zhu * and Li Wang \\ School of Traffic and Transportation, Beijing Jiaotong University, Beijing 100044, China; \\ 17114203@bjtu.edu.cn (Q.Z.); liwang@bjtu.edu.cn (L.W.) \\ * Correspondence: xnzhu@bjtu.edu.cn
}

Received: 13 February 2019; Accepted: 26 March 2019; Published: 1 April 2019

\begin{abstract}
Track allocation optimization in railway stations is one of the most fundamental problems for scheduling trains, especially in multi-direction high-speed railway stations. With the construction of high-speed rail networks, this kind of station has become increasingly common. However, the track allocation depends not only on the station tracks, train timetable, and rolling stock plan, but also on the resources in the station throat area. As a result, an effective track allocation plan becomes significant but also difficult. In this paper, we consider all these factors to make the results more practicable and an integer linear model that minimizes the total occupation time of resources in the throat area is presented. A flexible track utilization rule is also adopted to this model to fit the characteristics of the multi-direction station. Meanwhile, a detailed explanation of resources' occupation time is illustrated to facilitate the representation of the conflicting constraints. To resolve these issues, we use a commercial solver with its default parameters. A computational experiment of a station is conducted to verify the effectiveness of the proposed model. The resources utilization plan indicates that the capacity of a station is limited by the throat area, rather than by the station tracks.
\end{abstract}

Keywords: transportation; track allocation; resource utilization; train platforming; train control; integer linear programming

\section{Introduction}

High-speed rail has the advantages of safety, speed, high-reliability, and convenience. It is a good choice for passengers traveling between two countries. Developing a high-speed railway system has been seen as an important development direction for transportation in China. With the construction of high-speed rail in China, the length of high-speed rail was already approximately $29,000 \mathrm{~km}$ at the end of 2018. According to a plan issued by the Chinese government, this will be increased to $30,000 \mathrm{~km}$ by 2020 and to $38,000 \mathrm{~km}$ by 2025, making China one of the most advanced countries in the world in high-speed railway technology.

The track allocation problem, also known as the train platforming problem (TPP), is to safely assign the station's resources to the trains scheduled in the train diagram. The most important resources in stations include not only the siding tracks and main tracks near the platforms, but also the switches in the throat area. The high frequency and short dwelling time of trains in the high-speed railway stations, especially the multi-direction stations where the resources utilization is more complicated, make the problem more difficult than for conventional railway stations. Most track allocation planning is currently done manually and this method is error prone and always takes a long time to find the result. To improve the capacity of high-speed railway system, it is not generally the first choice to re-construct or enlarge the stations. Enhancing the quality of track allocation plan will be significant because it will save lots of investment, which can be used to improve rail freight services, as occurs in Indonesia [1]. 
The high-speed rail is independent of conventional rail in many countries, including China. Normally, more than two lines in the high-speed railway merge only in the station, which is described as a multi-direction station in this paper. Under the current management method, the timetables of different lines are designed one by one. Conflicts of train routes and platforms in connecting multi-direction stations are found and resolved 'by hand'. With the rapid construction of high-speed rail in recent years, the number of these stations has increased constantly, making the coordination in multi-direction stations more and more difficult. Meanwhile, the timetable plan and the track allocation plan of high-speed railway are designed iteratively. If there is no feasible track allocation solution under the current timetable plan, the timetable plan should be re-designed. Consequently, the challenge of how to obtain a track allocation plan in a multi-direction high-speed railway station effectively and efficiently is an urgent problem to be resolved.

Two kinds of layouts of multi-direction stations exist in the network. The first case is the station where main tracks of two lines are separated into two yards and the second is the station where main tracks merge into one yard, as shown in Figure 1. The first layout has no difference from the single-direction station and the corresponding allocation problem is much easier to address. However, conflicts between different trains are much more likely to happen in the second layout. Meanwhile, Marinov et al. (2013) showed that the second case is a common version for crossing stations [2]. Thus, the second case is selected and will be addressed in this paper.
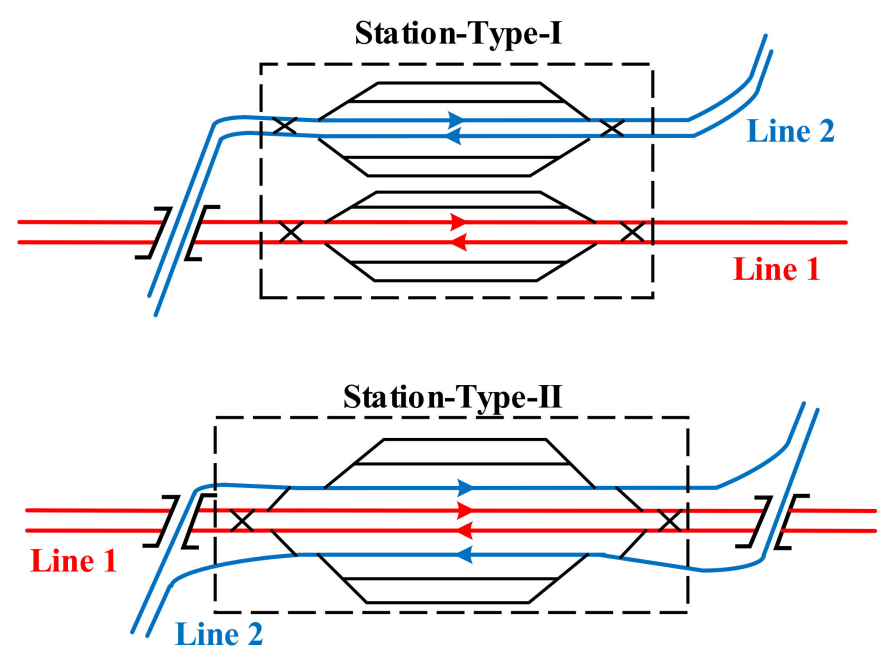

Figure 1. Two main kinds of multi-direction stations.

Track allocation optimization, a subsequent problem to the train timetable problem, is one of the basic scheduling problems for railway operations and has attracted lots of research. Rail management levels are divided into three levels: strategic, tactical and operational. The strategic level emphasizes the long term planning of company development. Timetables and schedules are developed in the tactical level. Operational level focuses on the rescheduling problem [2]. This paper mainly focuses on the tactical level.

Some researchers solved the train timetable problem from a microscopic perspective, including the track allocation constraints in their model. Meng and Zhou (2014) formulated a simultaneous rerouting and rescheduling model based on the time-space network [3]. The tracks in the network were divided into lots of cells. The proposed model stipulated that one train could traverse one cell once. However, some trains in the multi-direction station, for example turnaround trains, occupy the same resource more than once. Gao et al. (2017) integrated the station track assignment into their basic model to address the additional trains' scheduling problem. They assumed inbound and outbound trains are independent in the rail corridor [4]. This simplification was not desirable in this paper because the connections between trains from different directions are complicated in the multi-direction station and are not independent. 
Meanwhile, many researchers have studied the train platforming problem and several methods were used. The most common approaches are the graph coloring approach and the node packing approach. Cardillo et al. (1998) proposed a graph coloring approach which assigned different colors (tracks) to trains with conflicting routes [5]. Billionnet (2003) proposed two integer linear programs based on this method [6]. Zwaneveld et al. (2001) formulated the node packing method which defined conflict-free routes using the preprocessing technique [7]. Lusby et al. (2011) formulated the problem as a set-packing model with a resource-based constraint system, which was proven to be tighter than the conventional node-packing model [8]. Carey et al. (2003) used the simulation method, a scheduling algorithm that integrated the manual method presented [9].

In recent years, with the development of computer technology and some convenient software, researchers preferred to construct an integer programming model to address this problem. Qiao (2008) studied the selection and adjustment of routes in a high-speed railway station and proposed a multi-objective optimization model [10]. Based on Qiao (2008), Liu (2017) addressed the track allocation problem under abnormal conditions [11]. Wu et al. (2013) used a mean-variance model and optimized the variance of each resource [12]. However, these researchers did not fully consider the resources in the throat area. Sels et al. (2014) illustrated the occupation time of different resources, but they ignored the fact that the train should claim the resources before its actual occupation [13].

The contribution of this paper is described as follows:

- Firstly, to our knowledge, there is litte research that directly pays attention to the track allocation optimization in a multi-direction high-speed railway station. We propose the track allocation problem in the multi-direction high-speed railway station for the first time.

- Secondly, the resources in the throat area are considered. Some researchers assumed that outbound and inbound trains are independent in the single-direction station and separated the track allocation plan into two parts. In this way, headway constraints in the train timetabling problem can make sure that no conflict exists between routes of the trains from the same line in a single-direction station. However, in the multi-direction station, trains' arrival directions and departure directions are various. Train timetables of different lines are designed separately. The routes of trains from different lines may conflict with each other due to the layout of the throat area. Consequently, we cannot divide the track allocation plan into two independent parts based on their directions. The flexible track utilization rule is applied to this problem and all trains are allocated together. The restrictive resources of one multi-direction station are the switches in the throat area, and the track allocation plan is unfeasible if only the tracks near the platforms are considered. In this paper, the resources in the throat area and arrival-departure tracks are considered simultaneously and outbound and inbound trains are no longer independent in the station, which ensures the feasibility of the result.

- Thirdly, the resources occupation times were not explained exhaustedly and correctly, which lead to a difficult expression of the constraints when solving the track allocation problem of multi-direction stations. We give a detailed analysis of the occupation times of resources in the throat area and arrival-departure tracks in this paper, which facilitates the construction of the complicated conflicting constraints.

The remainder of the paper is organized as follows. In the next section, we give a detailed description of the track allocation problem in the multi-direction high-speed railway station. Then, an integrated optimization model is formulated and solved by a commercial solver with its default parameters. Finally, a computational experiment is conducted to verify the effectiveness of the proposed model and conclusions and recommendations for future research are given.

\section{Problem Description}

In this section, we will give a detailed problem description. In the first part, we will represent the physical environment of the problem in this paper. In the second part, the occupation time of the 
arrival-departure tracks and resources in the throat area will be illustrated. Finally, several assumptions in this paper are summarized.

\subsection{Multi-Direction Stations in a High-Speed Railway Network}

A multi-direction station is a station located on more than one railway line. We give an illustration of how two lines merge in a multi-direction station and the detailed station layout of the second case mentioned above as Figure 2. For the high-speed rail, the lines are all equipped with double-track. The considered multi-direction stations are generally symmetrical and two lines are separated by the bridge outside the station.

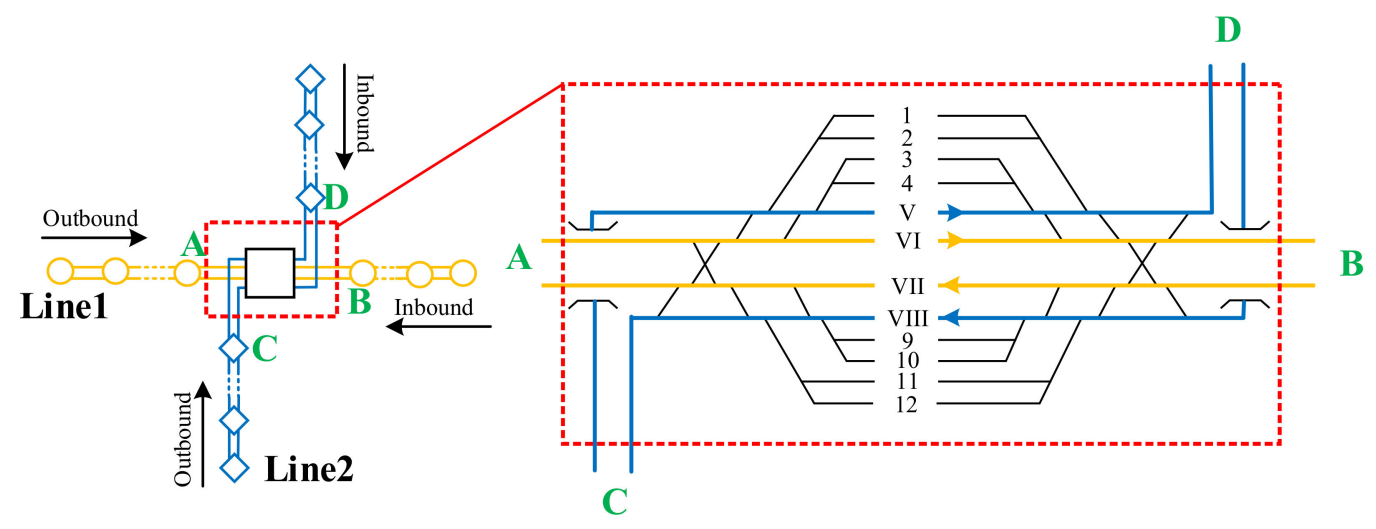

Stations on Line $1 \diamond$ Stations on Line $2 \square$ Station on Lines 1 and 2

Figure 2. Layout of multi-direction station located in two lines.

Tracks in a station can be separated into two parts: the main tracks and siding tracks. Main tracks, also known as main lines, are tracks that directly traverse the station, like blue and yellow lines in Figure 2. Main tracks in this station are used for nonstop trains to traverse the station. Siding tracks usually located next to the main tracks and are used for trains to dwell so that passengers can board or alight. The usage of main tracks is fixed according to trains' arrival and departure directions. However, the utilization of the siding tracks in the station is flexible, which means the inbound and outbound stop trains are dependent in the station and can be assigned to any siding tracks connected with their directions. As shown in Figure 2, stop trains from A to B (D) can be assigned not only to tracks $1,2,3$, and 4 , but also to tracks $9,10,11$, and 12 . This rule is called the flexible track utilization rule. In contrast, if those trains from A can only be assigned to tracks 1,2,3, and 4, this rule is called the independence rule. The existing studies mainly focused on the independence rule and separated the track allocation plan into two parts: an outbound track allocation plan and an inbound track allocation plan $[4,12,14]$. This independence rule reduces the complexity of the problem, however, it may obtain an unfeasible solution when dealing with track allocation problem in multi-direction station. In Figure 2, trains running between direction $B(A)$ and direction $D(C)$ and the turnaround trains, such as trains from direction A (C) to direction A (C), cannot be assigned to the tracks in a station under the independence rule. Thus, the independence rule is not applicable to the station discussed in this paper. Consequently, the flexible track utilization rule, rather than the independence rule, is applied in the multi-direction station.

The trains' operations in a station can be divided into three categories: the receiving train operations, the train static operations on the siding tracks and the departing train operations. Train operations in the railway station are controlled by the signal system and interlocking system. The train static operations on the siding tracks include water-supplying and sewage-suction. The train moving operations (i.e., receiving and departing operations) in a station are denoted by the routes. 


\subsection{Occupation Time of Train Routes}

Different trains' movements are governed by different conflict-free routes. The routes in a station includes the receiving routes, the departure routes and the through routes. The first two types of routes control the stop train's movement while the last type of routes controls the nonstop trains' moving operation. The receiving routes for stop trains start from the home signal, and end at the train stop point. The departure routes for stop trains start from the train stop points and end at the reverse home signal (the home signal for the opposite trains). The through routes are from the home signal to the reverse home signal shown as Figure 3 [15].

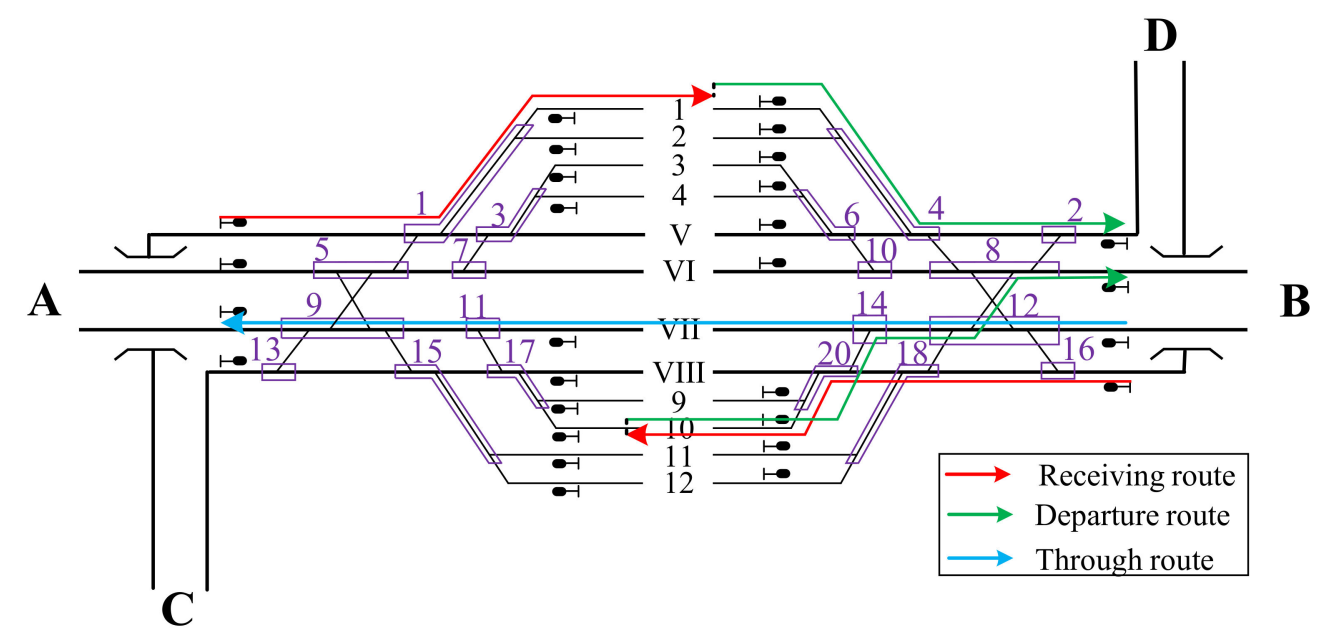

Figure 3. Train routes in the station.

The main resources in the throat area are the switches. The switches in the throat area can be divided into different groups (SG). Different routes will occupy different group combinations of switches. For example, the receiving route from direction $C$ to track 1 claims SG1 while the departure route from track 10 to direction B occupies SG20, SG14, SG12 and SG8 shown as Figure 3. Two routes that occupy different SGs are called parallel routes. Two routes which claim the same SG at the same time are called conflicting routes and cannot occupied by two trains at the same time.

The moving operation mainly occurs on the tracks of the throat area. In practice, the movements from one direction to one track or from one track to one direction generally occupy some determined SGs. For example, the basic route for movement from track 10 to direction B occupies SG20, SG14, SG12 and SG8, rather than SG20, SG18, SG12 and SG8.

Current studies give an explanation of some important time points when a train traverses a station $[13,15]$. In the station interlocking system, the resources of each route have been claimed by trains before their actual occupation. To the best of our knowledge, this fact was ignored in the studies of the track allocation problem $[5,6,13]$. Our previous research put forward this argument, but we adopted the independence track utilization rules. We assumed the outbound and inbound trains are independent and separated the track allocation plan of one station into two parts: an outbound plan and an inbound plan to reduce the complexity of the problem [15]. In this paper we adopt the flexible track utilization rules rather than independence, making the solution more practical.

For a stop train, the resources of the receiving route in the throat area and the corresponding tracks will be claimed under the minimum receiving preparation time criterion before the arrival time given by the train diagram. When the tail of the train passes by the reverse starting signal (starting signal for the opposite direction train of the same siding track), the resources of the train's receiving route in the throat area will be released after the buffer time and then are available for other trains. The departure route will also be locked to the minimum departure preparation time criterion before the train's departure time. When the tail of the train passes the starting signal, the siding tracks will be 
available for other trains after the buffer time. The resources of the train's departure route in the throat area are unlocked in buffer time after the tail of the train passing by the reverse home signal.

For a nonstop train, the occupation time of resources is slightly different from the stop train shown as Figure 4. The SGs in the departure part are claimed once the through route of the nonstop train is prepared. The main tracks can only be claimed by the nonstop trains. Thus, the occupation time of the main track is synchronous with the SGs in the departure part.

Resources Occupation time of Stop Trains

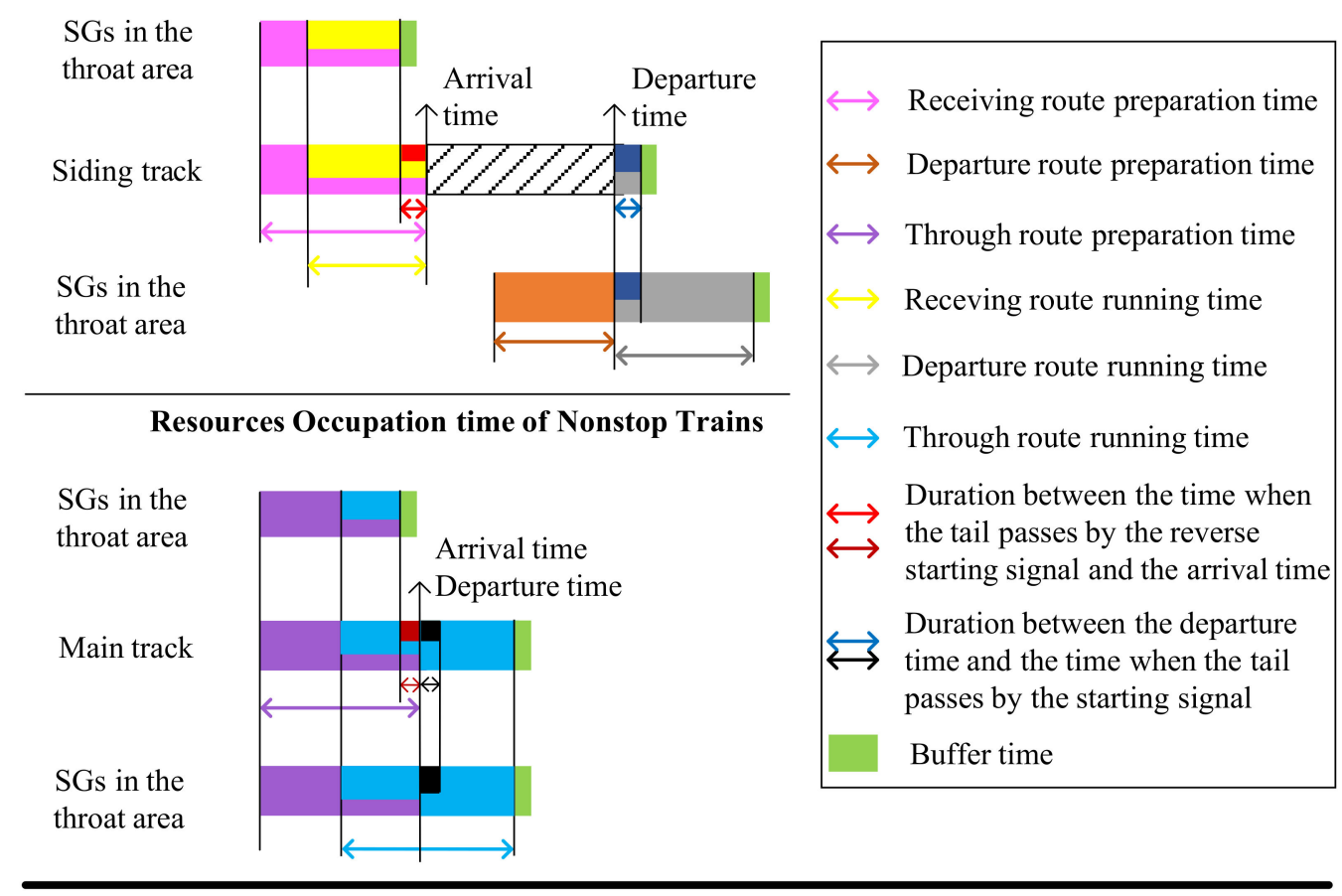

Figure 4. Stop and nonstop trains' occupation time of the resources.

Trains traversing a station should be assigned with conflict-free resources. The occupation time of one resource should be as short as possible so that the resources can be available to other trains, especially in the multi-direction station where the conflicting route happens frequently. Consequently, we minimize the sum of the occupation time of different resources in the objective function.

\subsection{Assumption}

To elaborate the track allocation problem in the multi-direction high-speed railway station, the assumptions made in this paper are summarized as follows:

1. The second type of the station layout mentioned above is discussed.

2. Basic information of the station is given, including the minimum preparation time criterion and running time of different routes and the operations each track can provide.

3. The speed differences in a station only exist between stop and nonstop trains. Because of the speed limitation in the station, the running time of nonstop trains are the same, regardless of the train types and the speed levels. The same assumption is applied to the stop trains.

4. The train timetable and rolling stock plan are given, including the trains' static operation in this multi-direction station.

5. The basic receiving route from one arrival direction to one certain track is unique and fixed. Similarly, the basic departure route is also definite and unique. 
The first assumption has been explained. The preparation time criteria and the operations each track can provide are stipulated in the corresponding station documents. The running time of different routes can be collected from daily operation. The running time of trains in the links between stations depends on train types and speed level, however, the speeds of all stop trains in the station are almost the same. Meanwhile, due to the short length of the main tracks in the station, the running time of nonstop trains with different speeds is not significant. The planning process of the high-speed railway shows that the train timetable and rolling stock plan are the input to the track allocation planning [15]. The train timetable shows the arrival and departure time of trains, and the rolling stock plan represents which two trains share one rolling stock and should be assigned to one track.

\section{Formulation of Optimization Model}

In this section, an integer linear optimization model is formulated for the track allocation problem in multi-direction high-speed railway stations. We will give the notations, the decision variables, the constraints and the objective function of the model.

\subsection{Notations and Decision Variables}

$I_{N}=$ set of trains scheduled to be nonstop trains in this station;

$I_{S T}=$ set of trains scheduled to be stop trains in this station;

$I=$ set of trains that traverse this station. $I=I_{N} \cup I_{S T}, i, k \in I$ and $|I|=n$.

$J_{N}=$ set of main tracks in this station;

$J_{S T}=$ set of siding tracks in this station;

$J=$ set of tracks in this station. $J=J_{N} \cup J_{S T}, j, s \in J$ and $|J|=m$.

$t_{i}^{p a}=$ time point when the receiving route is prepared for train $i$ in this station;

$t_{i}^{p d}=$ time point when the departure route is prepared for train $i$ in this station;

$t_{i}^{a}=$ time point when train $i$ arrives at this station. This time point is given by the train timetable.

$t_{i}^{d}=$ time point when train $i$ departs from this station. This time point is given by the train timetable.

$t_{i}^{a d s}=$ the time when train $i$ starts to occupy the arrival-departure track.

$t_{i}^{a d e}=$ the time when train $i$ ends up occupying the arrival-departure track.

$t_{i j v_{i}^{d}}^{d e}=$ the time when the SGs occupied by the departure route of train $i$ from track $j$ to direction $v_{i}^{d}$

are available for other trains.

$t_{i v_{i}^{a} j}^{a}=$ the time when the SGs occupied by the receiving route of train $i$ from direction $v_{i}^{a}$ to track $j$ are available for other trains.

$v_{i}^{a}=$ arrival direction of train $i$ in this station.

$v_{i}^{d}=$ departure direction of train $i$ in this station.

$T_{\text {min }}^{p a}=$ minimum time difference criterion between the time point $t_{i}^{p a}$ and the time point $t_{i}^{a}$.

$T_{\text {min }}^{p d}=$ minimum time difference criterion between the time point $t_{i}^{p d}$ and the time point $t_{i}^{d}$.

$T_{N}^{a e}=$ time difference between the time point when the tail of a nonstop train passes by the reverse starting signal and its arrival time.

$T_{S T}^{a e}=$ time difference between the time point when the tail of a stop train passes by the reverse starting signal and its arrival time.

$T_{S T}^{a d e}=$ time difference between the stop train's departure time and the time when the tail of the train passes by the starting signal.

$T_{j v_{i}^{d}}=$ running time of the departure routes.

$T_{b u f}=$ duration time of one resource recovered from its locked status to released status.

$\varphi\left(v_{i}^{a}, v_{i}^{d}\right)=$ set of tracks that can be used for train $i$ from direction $v_{i}^{a}$ to direction $v_{i}^{d}$.

$r\left(v_{i}^{a}, j\right)=$ set of SGs that will be used for train $i$ from direction $v_{i}^{a}$ to track $j$.

$r\left(j, v_{i}^{a}\right)=$ set of SGs that will be used for train $i$ from track $j$ to direction $v_{i}^{d}$. 
$\beta_{i}^{o p}=\in\{0,1\}, \beta_{i}^{o p}=1$, if train $i$ should be provided with a certain static operation op; $\beta_{i}^{o p}=0$, otherwise.

$\gamma_{j}^{o p}=\in\{0,1\}, \gamma_{j}^{o p}=1$, if track $j$ can provide a certain static operation $o p ; \gamma_{j}^{o p}=0$, otherwise.

The propose of the track allocation problem is to decide which track in the station train $i$ should be assigned to.

$x_{i}^{j}=\in\{0,1\}, x_{i}^{j}=1$, if track $j$ is assigned to train $i ; x_{i}^{j}=0$, otherwise.

\subsection{Objective Function}

As is stated in section 'Occupation time of train routes', the objective function is to minimize total trains' occupation time of resources in the throat area. The occupation time of the SGs of the receiving route is fixed for each train and will not change with the tracks the trains are allocated to. Generally, the minimum preparation time criterion is larger than the running time of the receiving route. As a result, the occupation time of the receiving routes depends on the minimum preparation time criterion. Thus, this objective function can be reduced to minimize total trains' occupation time of the resources on the trains' departure routes.

$$
\min f=\sum_{j \in J} \sum_{i \in I} x_{i}^{j} \cdot T_{j v_{i}^{d}}
$$

The balanced usage of tracks is adopted as the objective function in lots of research on the track allocation problem in single-direction stations and makes the optimization model nonlinear. However, after interviewing twelve experienced dispatchers in the most advanced Beijing-Shanghai high-speed railway in China, the benefits of the balanced usage are found to not be worth the complexity of nonlinearity in the multi-direction station. The number of feasible solutions to the track allocation problem of multi-direction station is very limited. The objective of this problem should be as simple as possible. Consequently, we do not consider other nonlinear objective functions. At the same time, the objective of this model is to make sure this model can be used to determine the minimum number of arrival-departure tracks at the station design stage.

\subsection{Constraints}

For clarity, the constraints of the track allocation problem can be divided into several parts, namely time constraints, safety constraints, operation constraints and domain constraints.

\subsubsection{Time Constraints}

$$
\begin{gathered}
t_{i}^{p a}=t_{i}^{a}-T_{m_{i n}{ }^{p}}^{p a}, \forall i \in I_{S T} \\
t_{i}^{p d}=t_{i}^{d}-T_{m i n^{\prime}}^{p d}, \forall i \in I_{S T} \\
t_{i v_{i}^{a} j}^{a a}=t_{i}^{a}-T_{S T}^{a e}+T_{b u f}, \forall i \in I_{S T}, j \in \varphi\left(v_{i}^{a}, v_{i}^{d}\right) \\
t_{i}^{a d s}=T_{i}^{p a}, \forall i \in I_{S T} \\
t_{i}^{a d e}=t_{i}^{d}+T_{S T}^{a d e}+T_{b u f}, \forall i \in I_{S T} \\
t_{i j v_{i}^{d e}}^{d e}=t_{i}^{d}+T_{j v_{i}^{d}}+T_{b u f}, \forall i \in I_{S T}, j \in \varphi\left(v_{i}^{a}, v_{i}^{d}\right) \\
t_{i}^{p a}=t_{i}^{a}-T_{m i n^{\prime}}^{p a}, \forall i \in I_{N} \\
t_{i}^{p d}=t_{i}^{p a}, \forall i \in I_{N} \\
t_{i j v_{i}^{d}}^{d e}=t_{i}^{d}+T_{j v_{i}^{d}}+T_{b u f}, \forall i \in I_{N}, j \in \varphi\left(v_{i}^{a}, v_{i}^{d}\right)
\end{gathered}
$$




$$
\begin{gathered}
t_{i v_{i}^{a} j}^{a e}=t_{i}^{a}-T_{N}^{a e}+T_{b u f}, \forall i \in I_{N}, j \in \varphi\left(v_{i}^{a}, v_{i}^{d}\right) \\
t_{i}^{a d s}=T_{i}^{p a}, \forall i \in I_{N} \\
t_{i}^{a d e}=t_{i j v_{i}^{d}}^{d e}, \forall i \in I_{N}
\end{gathered}
$$

Constraints (2)-(7) are related to the track occupation time points of the stop trains. Constraints (8)-(13) define the track allocation time points of the nonstop trains. These constraints enforce the trains' occupation time windows. Each train has to be assigned to a conflict-free route during its time window. Time constraints are the prerequisites for subsequent constraints and are proposed based on the detailed analysis of the resources' occupation time in this paper.

\subsubsection{Safety Constraints}

$$
\begin{gathered}
x_{i}^{j}+x_{k}^{j} \leq 1, \forall i, k \in I, j \in J,\left(t_{i}^{a d s}, t_{j}^{a d e}\right) \cap\left(t_{k}^{a d s}, t_{k}^{a d e}\right) \neq \phi \\
x_{i}^{j}+x_{k}^{s} \leq 1, \\
\forall i, k \in I, \forall j, s \in J, r\left(v_{i}^{a}, j\right) \cap r\left(v_{k^{\prime}}^{a}, s\right) \neq \phi,\left(t_{i}^{p a}, t_{i v_{i}^{a} j}^{a e}\right) \cap\left(t_{k}^{p a}, t_{k v_{k}^{a} a_{s}}^{a e}\right) \neq \phi \\
x_{i}^{j}+x_{k}^{s} \leq 1, \\
\forall i, k \in I, \forall j, s \in J, r\left(j, v_{i}^{d}\right) \cap r\left(s, v_{k}^{d}\right) \neq \phi,\left(t_{i}^{p d}, t_{i j v_{i}^{d}}^{d e}\right) \cap\left(t_{k}^{p d}, t_{k s v_{k}^{d}}^{d e}\right) \neq \phi \\
x_{i}^{j}+x_{k}^{s} \leq 1, \\
\forall i, k \in I, \forall j, s \in J, r\left(v_{i}^{a}, j\right) \cap r\left(s, v_{k}^{d}\right) \neq \phi,\left(t_{i}^{p a}, t_{i v_{i}^{a} j}^{a e}\right) \cap\left(t_{k}^{p d}, t_{k s v_{k}^{d}}^{d e}\right) \neq \phi
\end{gathered}
$$

Constraints 14 enforce the safety requirement of occupying the same track. If the arrival-departure track's occupation time of two trains overlap, these two trains cannot be assigned to one track. Constraints 14-16 make sure if two trains' occupation time of one resource overlap, these two trains will not be assigned to the tracks with the conflicting resources. Constraints 15 ensure that if two arrival trains' occupation time of SGs overlaps with each other, then two trains cannot be assigned to the tracks with the same SGs for arrival. Constraints 16 require that if two departure trains' occupation times of SGs overlap with each other, then two trains cannot be assigned to the tracks with the same SGs for departure. Constraints 17 are imposed on one arrival train and one departure train. These safety constraints are the basic constraints on the track allocation problem. Different researchers form these constraints from different perspectives. In this paper, the time window analysis and the time constraints make safety constraints easier to represent than existing studies. Constraints 15-17 represent that the track allocation and the resource utilization in the throat area are optimized simultaneously, which makes the result calculated by this model safe to adopt.

\subsubsection{Operation Constraints}

$$
\begin{gathered}
\sum_{j \in J_{S T}} x_{i}^{j} \gamma_{j}^{o p} \geq \beta_{i}^{o p}, \forall i \in I_{S T} \\
\sum_{j \in J_{N}} x_{i}^{j}=1, \forall i \in I_{N} \\
\sum_{j \in J_{S T}} x_{i}^{j}=1, \forall i \in I_{S T}
\end{gathered}
$$




$$
\sum_{j} x_{i}^{j}=0, \forall i \in I, j \notin \varphi\left(v_{i}^{a}, v_{i}^{d}\right)
$$

Constraints 18 represent that the train that need to be provided one static operation should be allocated to the tracks that can provide this operation. Constraints 19-20 show that nonstop trains must be assigned to main tracks and stop trains must be assigned to siding tracks, which ensures the feasibility of the track allocation plan. In practice, a train from one direction may not be able to be assigned to every track on account of the station layout. For example, the trains from direction $C$ cannot be allocated to tracks VI, VII, VIII, 9, 10, 11 and 12 in the station illustrated in Figure 3. Constraints 19-21 make the track utilization plan more acceptable from the station layout perspective.

\subsubsection{Domain Constraints}

$$
x_{i}^{j} \in\{0,1\}, \forall i \in I, \forall j \in J
$$

Constraints 22 define the domain of variables.

\section{Computational Experiment}

The proposed optimization model is preprocessed by the software MATLAB R2016b and then solved by the commercial solver IBM ILOG CPLEX 12.6.0 with its default parameters. Our computational experiments are run on a computer with Intel (R) Core (TM) i5-7200U $2.50 \mathrm{GHz}$ and 4.00 GB memory and Microsoft Windows 10 (64 bit) operation system.

The layout of the multi-direction high-speed railway station in the computational experiment is illustrated above (Figure 3). The timetable of 49 trains during peak hours between 12 a.m. and 2 p.m. in this station and several basic station data are collected from the station documents and historical records (Tables 1-3). The arrival time and departure time of the turnaround train are obtained by analyzing the given timetable and the rolling stock plan. For example, train 43 from direction A to direction $\mathrm{A}$ is actually separated into two trains in the original timetable (Table 1). However, the rolling stock plan shows these two trains share one rolling stock and should be assigned to one track, otherwise, the track allocation plan will be invalid. In this case, the trains' timetable is updated (Table 1).

Table 1. Train Timetable of All Trains in the Station.

\begin{tabular}{cccccc}
\hline Train ID & $\begin{array}{c}\text { Arrival } \\
\text { Direction }\end{array}$ & $\begin{array}{c}\text { Departure } \\
\text { Direction }\end{array}$ & Arrival Time & $\begin{array}{c}\text { Departure } \\
\text { Time }\end{array}$ & $\begin{array}{c}\text { Stop (1) or } \\
\text { Nonstop (0) }\end{array}$ \\
\hline 1 & D & A & $12: 00: 00$ & $12: 03: 20$ & 1 \\
2 & B & A & $12: 02: 00$ & $12: 06: 24$ & 1 \\
3 & B & A & $12: 05: 10$ & $12: 11: 50$ & 1 \\
4 & C & D & $12: 06: 20$ & $12: 12: 20$ & 1 \\
5 & A & B & $12: 09: 40$ & $12: 09: 40$ & 0 \\
6 & B & A & $12: 09: 50$ & $12: 18: 20$ & 1 \\
7 & C & D & $12: 15: 00$ & $12: 17: 00$ & 1 \\
8 & D & C & $12: 16: 50$ & $12: 16: 50$ & 0 \\
9 & B & D & $12: 18: 10$ & $12: 24: 10$ & 1 \\
10 & D & C & $12: 20: 20$ & $12: 23: 20$ & 1 \\
11 & A & B & $12: 22: 00$ & $12: 22: 00$ & 0 \\
12 & C & A & $12: 26: 10$ & $12: 30: 10$ & 1 \\
13 & D & C & $12: 30: 00$ & $12: 33: 00$ & 1 \\
14 & C & D & $12: 30: 20$ & $12: 36: 00$ & 1 \\
15 & B & C & $12: 34: 30$ & $12: 36: 30$ & 1 \\
\hline
\end{tabular}


Table 1. Cont.

\begin{tabular}{|c|c|c|c|c|c|}
\hline Train ID & $\begin{array}{c}\text { Arrival } \\
\text { Direction }\end{array}$ & $\begin{array}{l}\text { Departure } \\
\text { Direction }\end{array}$ & Arrival Time & $\begin{array}{l}\text { Departure } \\
\text { Time }\end{array}$ & $\begin{array}{l}\text { Stop (1) or } \\
\text { Nonstop (0) }\end{array}$ \\
\hline 16 & B & A & $12: 37: 47$ & $12: 41: 00$ & 1 \\
\hline 17 & $\mathrm{C}$ & $\mathrm{D}$ & $12: 38: 10$ & $12: 41: 10$ & 1 \\
\hline 18 & $\mathrm{D}$ & $\mathrm{C}$ & $12: 38: 30$ & $12: 42: 10$ & 1 \\
\hline 19 & $\mathrm{C}$ & $\mathrm{B}$ & $12: 43: 10$ & $12: 46: 10$ & 1 \\
\hline 20 & $\mathrm{D}$ & A & $12: 44: 30$ & $12: 51: 20$ & 1 \\
\hline 21 & B & A & $12: 45: 46$ & $12: 45: 46$ & 0 \\
\hline 22 & $\mathrm{C}$ & $\mathrm{D}$ & $12: 47: 00$ & 12:49:00 & 1 \\
\hline 23 & A & B & $12: 47: 10$ & $12: 51: 10$ & 1 \\
\hline 24 & $\mathrm{C}$ & $\mathrm{D}$ & $12: 50: 30$ & $12: 52: 45$ & 1 \\
\hline 25 & B & $\mathrm{C}$ & $12: 51: 00$ & $12: 55: 00$ & 1 \\
\hline 26 & A & B & $12: 52: 40$ & $12: 57: 00$ & 1 \\
\hline 27 & $\mathrm{C}$ & $\mathrm{D}$ & $12: 56: 00$ & $12: 58: 20$ & 1 \\
\hline 28 & B & A & $12: 56: 03$ & 13:00:03 & 1 \\
\hline 29 & $\mathrm{D}$ & $\mathrm{C}$ & $12: 57: 20$ & 13:01:46 & 1 \\
\hline 30 & A & $\mathrm{D}$ & 12:58:00 & 13:02:00 & 1 \\
\hline 31 & $\mathrm{D}$ & A & 13:03:00 & 13:06:20 & 1 \\
\hline 32 & $\mathrm{C}$ & $\mathrm{D}$ & 13:07:00 & 13:07:00 & 0 \\
\hline 33 & $\mathrm{D}$ & $\mathrm{C}$ & 13:07:00 & 13:10:00 & 1 \\
\hline 34 & B & $\mathrm{A}$ & $13: 08: 20$ & $13: 11: 20$ & 1 \\
\hline 35 & B & $\mathrm{C}$ & 13:13:10 & $13: 20: 10$ & 1 \\
\hline 36 & $\mathrm{D}$ & $\mathrm{C}$ & 13:16:00 & $13: 16: 00$ & 0 \\
\hline 37 & A & $\mathrm{D}$ & 13:17:00 & 13:19:00 & 1 \\
\hline 38 & B & A & $13: 18: 20$ & $13: 18: 20$ & 0 \\
\hline 39 & $\mathrm{D}$ & A & $13: 21: 00$ & $13: 26: 00$ & 1 \\
\hline 40 & $\mathrm{C}$ & B & $13: 25: 00$ & $13: 28: 00$ & 1 \\
\hline 41 & B & $\mathrm{C}$ & $13: 25: 00$ & $13: 28: 20$ & 1 \\
\hline 42 & B & $\mathrm{C}$ & $13: 28: 40$ & $13: 31: 20$ & 1 \\
\hline 43 & A & A & $13: 36: 10$ & $13: 48: 00$ & 1 \\
\hline 44 & $\mathrm{D}$ & $\mathrm{C}$ & $13: 40: 00$ & $13: 44: 10$ & 1 \\
\hline 45 & A & B & 13:41:00 & 13:43:00 & 1 \\
\hline 46 & B & A & $13: 42: 00$ & $13: 44: 00$ & 1 \\
\hline 47 & $\mathrm{C}$ & $\mathrm{D}$ & $13: 45: 00$ & $13: 47: 00$ & 1 \\
\hline 48 & $\mathrm{D}$ & B & $13: 48: 40$ & 13:53:00 & 1 \\
\hline 49 & A & $\mathrm{C}$ & $13: 54: 30$ & 13:59:00 & 1 \\
\hline
\end{tabular}

Table 2. Basic Time Data in the Station.

\begin{tabular}{ccccccc}
\hline$T_{\text {min }}^{p a}$ & $T_{\text {min }}^{p d}$ & $T_{N}^{a e}$ & $T_{S T}^{a e}$ & $T_{S T}^{a d e}$ & $T_{j v_{i}^{d}}$ & $T_{b u f}$ \\
\hline $180 \mathrm{~s}$ & $30 \mathrm{~s}$ & $2 \mathrm{~s}$ & $4 \mathrm{~s}$ & $8 \mathrm{~s}$ & $10 \mathrm{~s}$ & $10 \mathrm{~s}$ \\
\hline
\end{tabular}

Table 3. Running Time of Receiving and Departure Routes.

\begin{tabular}{cccccc}
\hline Arrival Direction & Track Number & $T_{v_{i}^{a} j}(\mathbf{s})$ & Track Number & Departure Direction & $T_{j v_{i}^{d}}(\mathbf{s})$ \\
\hline A & $1 / 2$ & 80 & $1 / 2$ & $\mathrm{~A}$ & 105 \\
$\mathrm{~A}$ & $3 / 4$ & 70 & $1 / 2$ & $\mathrm{~B}$ & 85 \\
$\mathrm{~A}$ & 6 & 10 & $1 / 2$ & $\mathrm{C}$ & 135 \\
$\mathrm{~A}$ & $9 / 10$ & 95 & $1 / 2$ & $\mathrm{D}$ & 50 \\
$\mathrm{~A}$ & $11 / 12$ & 105 & $3 / 4$ & $\mathrm{~A}$ & 95 \\
$\mathrm{~B}$ & $1 / 2$ & 105 & $3 / 4$ & $\mathrm{~B}$ & 80 \\
$\mathrm{~B}$ & $3 / 4$ & 95 & $3 / 4$ & $\mathrm{D}$ & 125 \\
$\mathrm{~B}$ & 7 & 10 & $3 / 4$ & $\mathrm{~B}$ & 45 \\
$\mathrm{~B}$ & $9 / 10$ & 70 & 5 & $\mathrm{D}$ & 20 \\
$\mathrm{~B}$ & $11 / 12$ & 80 & 5 & & 10 \\
\hline
\end{tabular}


Table 3. Cont.

\begin{tabular}{cccccc}
\hline Arrival Direction & Track Number & $\boldsymbol{T}_{v_{i}^{a} j}(\mathbf{s})$ & Track Number & Departure Direction & $T_{j v_{i}^{d}}(\mathbf{s})$ \\
\hline C & $1 / 2$ & 40 & 6 & B & 10 \\
C & $3 / 4$ & 35 & 6 & $\mathrm{D}$ & 20 \\
$\mathrm{C}$ & 5 & 10 & 7 & $\mathrm{~A}$ & 10 \\
$\mathrm{D}$ & $1 / 2$ & 135 & 7 & $\mathrm{C}$ & 20 \\
$\mathrm{D}$ & $3 / 4$ & 125 & 8 & $\mathrm{~A}$ & 20 \\
$\mathrm{D}$ & 8 & 10 & 8 & $\mathrm{C}$ & 10 \\
$\mathrm{D}$ & $9 / 10$ & 35 & $9 / 10$ & $\mathrm{~A}$ & 80 \\
$\mathrm{D}$ & $11 / 12$ & 40 & $9 / 10$ & $\mathrm{~B}$ & 95 \\
- & - & - & $9 / 10$ & $\mathrm{C}$ & 45 \\
- & - & - & $9 / 10$ & $\mathrm{D}$ & 125 \\
- & - & - & $11 / 12$ & $\mathrm{~A}$ & 85 \\
- & - & - & $11 / 12$ & $\mathrm{~B}$ & 105 \\
- & - & - & $11 / 12$ & $\mathrm{C}$ & 50 \\
- & - & - & $11 / 12$ & $\mathrm{D}$ & 135 \\
\hline
\end{tabular}

\section{Results}

The proposed optimization model is pre-processed by the software MATLAB R2006b and then solved by the commercial solver IBM ILOG CPLEX 12.6.0 with the branch and cut method. Finally, we obtain the track allocation plan. Unlike current research, we represent the track occupation time of all trains rather than the arrival and departure time (Figure 5). To verify the model, we also give the GSs' occupation plan (Figure 6), where black blocks represent the resources that are occupied.

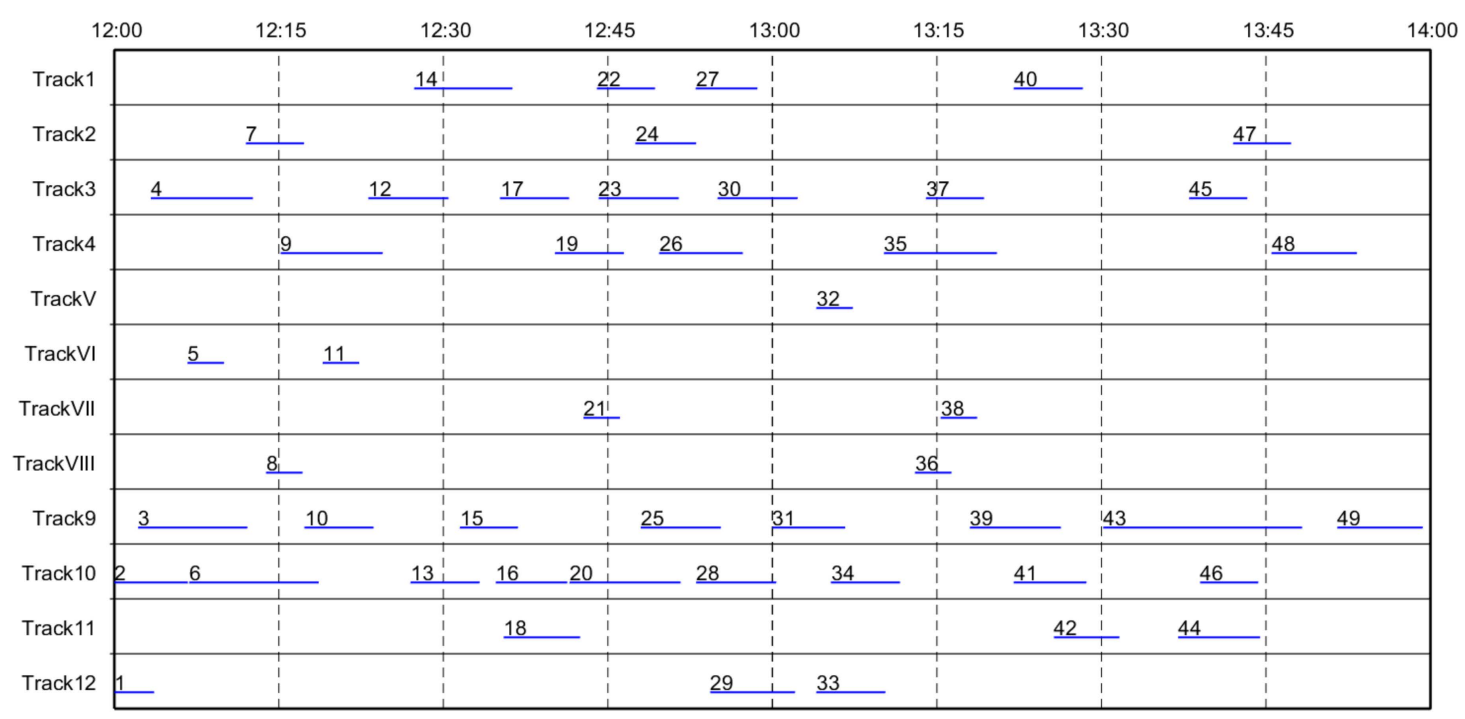

Figure 5. Track allocation time by all trains. 


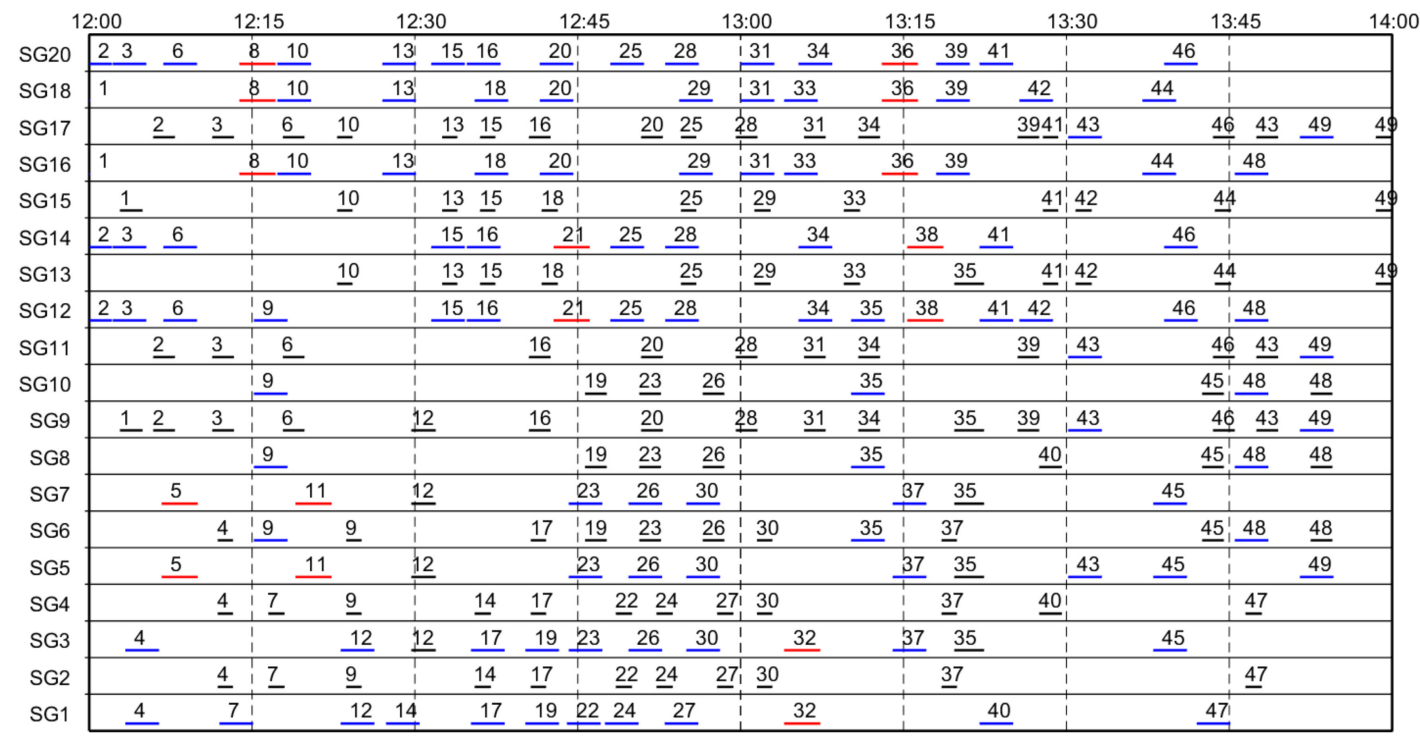

Figure 6. Occupation time of SGs in the station. Blue line: occupation of receiving routes. Black line: occupation of departure routes. Red line: occupation of through routes.

Clearly, all the nonstop trains are assigned to the main tracks and all the stop trains are allocated to the siding tracks, which satisfies the constraints. The siding tracks, main tracks and SGs are occupied by all trains without conflict, which verifies the proposed model is valid.

Train 35 is allocated to the track 4 under the flexible track utilization rule, which makes the value of objective function larger than the allocation if it is assigned to tracks 9-12. However, in that case, the receiving route of train 35 will conflict with others. Meanwhile, trains 9, 12, 43, 48 and 49 are turnaround trains, which cannot get a feasible solution without the flexible track utilization rule.

To compare the allocation time of different resources, we analyze every track time utilization rate (Figure 7) and every SG time utilization rate (Figure 8).

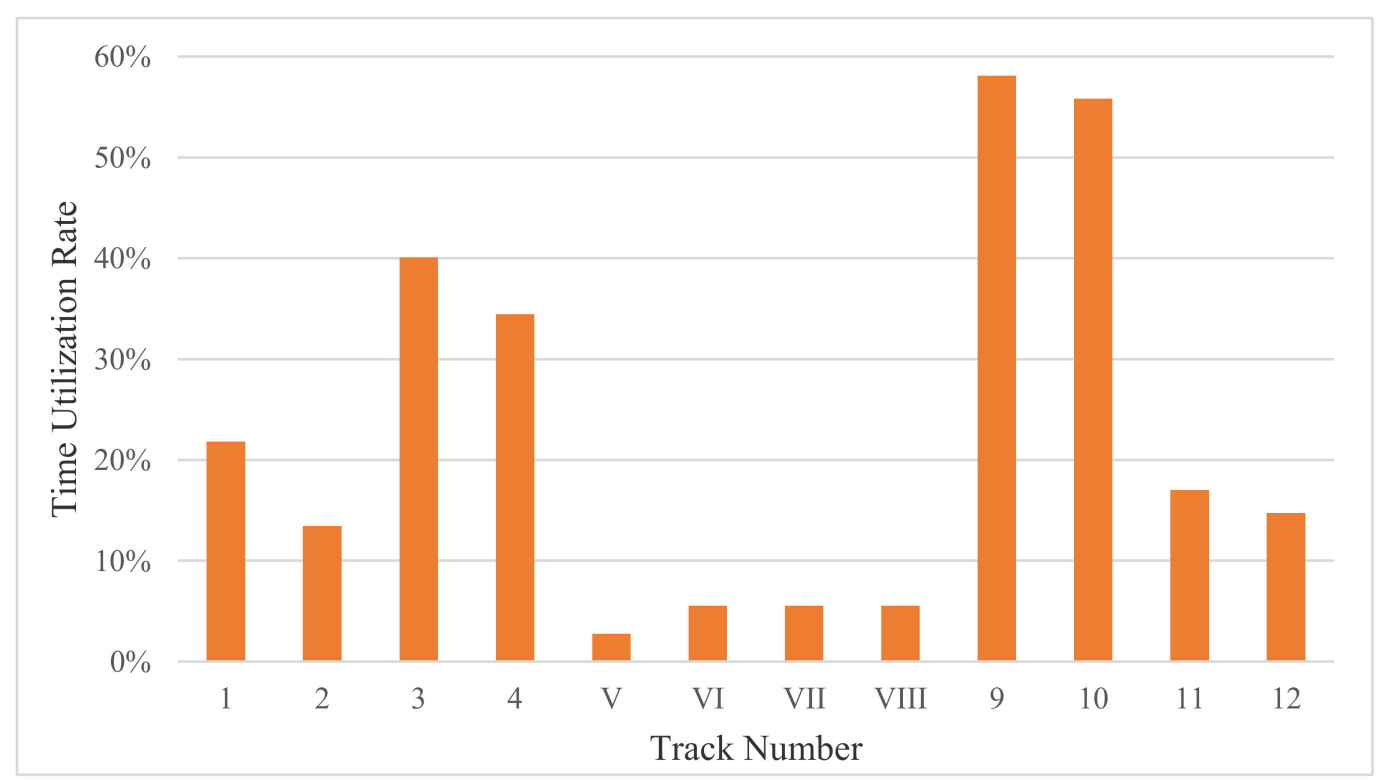

Figure 7. Time utilization rate of every track. 


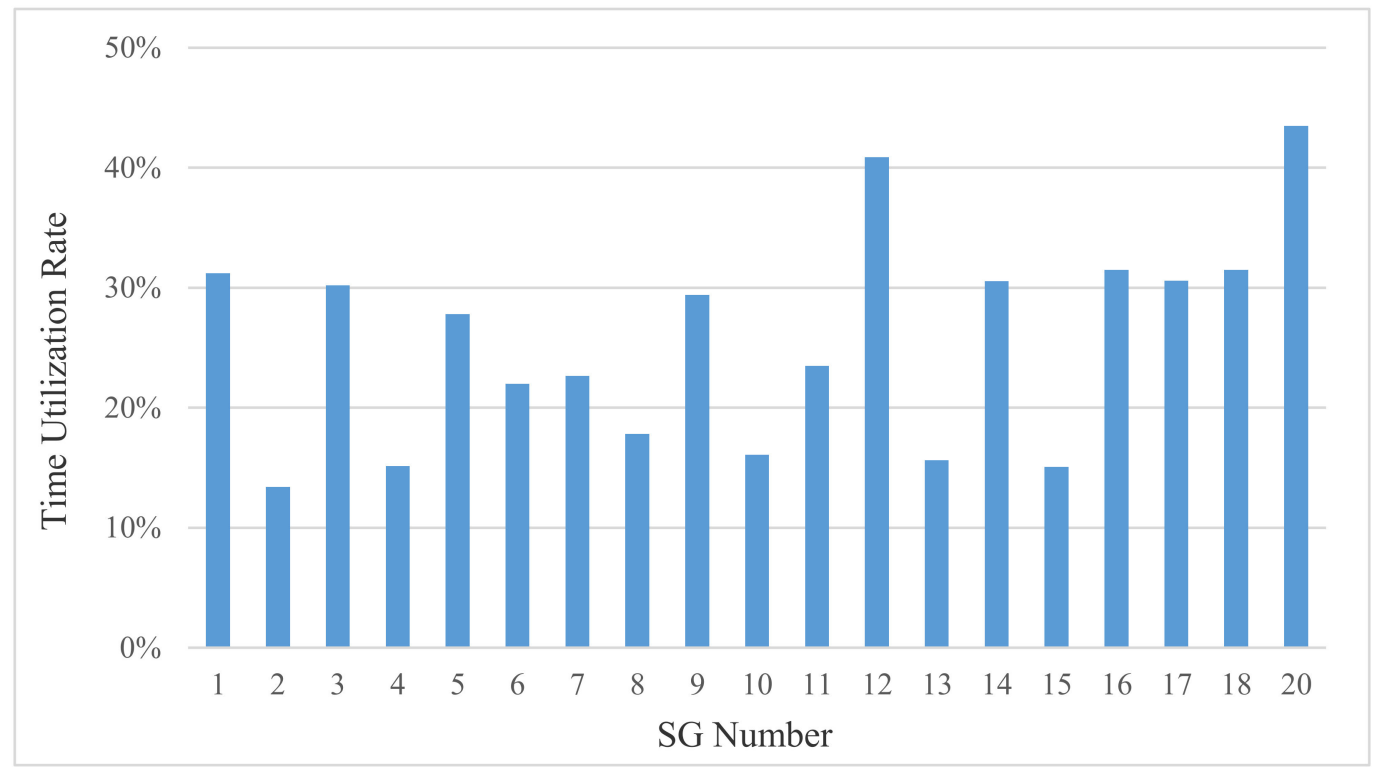

Figure 8. Time utilization rate of every SG.

For more than $50 \%$ of tracks, the time utilization rates are between $0 \%-20 \%$. In contrast, for most SG, time utilization rates are between 10\%-30\%. In addition, the expectation value of time utilization rates of tracks and SG are $22.9 \%$ and $25.7 \%$ respectively. Meanwhile, we compared the track allocation time with the SGs' occupation time, the SGs in this multi-direction high-speed railway station are occupied more frequently with the increase of the number of trains from 12:30 to 13:30. This denotes that the station capacity depends on the capacity of throat area rather than station tracks. Thus, simultaneous optimization of the usage of arrival-departure tracks and resources in the throat area is significant to multi-direction stations.

\section{Conclusions}

High-speed rail is a system to transit passengers, which is really different from rail freight in its technical aspects. The frequency of trains in high-speed railway stations, especially in multi-direction stations, is much higher than that of rail freight stations. As a result, train operations are more frequent and the conflicts of different trains' routes may occur more frequently in studied stations than in rail freight stations. It is therefore much more important for multi-direction high-speed railway stations to allocate trains to the tracks with a higher quality method. The resolution of conflicts in high-speed railway stations is currently done 'by hand'. Our proposed model is more practical and easier to use to generate a feasible track allocation plan of multi-direction high-speed railway stations as illustrated in this paper without conflicts of resources in both arrival-departure tracks and the throat area, which can be used to support the planning decisions in the future.

In this paper, the problem of track allocation in a multi-direction high-speed railway station is studied. Because the trains' directions vary, we apply the flexible track utilization rule and assign the resources in the station to different trains simultaneously. The flexible track rule rather than the independence rule guarantees that a feasible track allocation plan of the multi-direction station is obtained. The comparison of this paper with other classical papers on the track allocation problem is summarized in Table 4. 
Table 4. The comparison of this paper with other papers.

\begin{tabular}{|c|c|c|c|c|c|c|}
\hline Publication & $\begin{array}{l}\text { Arri./Dep. } \\
\text { Route }^{1}\end{array}$ & $\begin{array}{c}\text { Occupation } \\
\text { Time Analysis } 2\end{array}$ & Pre-acclaim ${ }^{3}$ & $\begin{array}{l}\text { Flexible } \\
\text { Rules }{ }^{4}\end{array}$ & Multi-direction $^{5}$ & $\begin{array}{c}\text { Rolling } \\
\text { Stock Plan } 6\end{array}$ \\
\hline Meng and Zhou (2014) & $\mathrm{N}$ & $\mathrm{N}$ & $\mathrm{N}$ & - & - & $\mathrm{N}$ \\
\hline Gao et al. (2017) & $\mathrm{N}$ & $\mathrm{N}$ & $\mathrm{N}$ & $\mathrm{N}$ & $\mathrm{N}$ & $\mathrm{N}$ \\
\hline Billionnet (2003) & $\mathrm{N}$ & $\mathrm{N}$ & $\mathrm{N}$ & - & - & $\mathrm{N}$ \\
\hline Sels et al. (2014) & $\mathrm{Y}$ & $\mathrm{Y}$ & $\mathrm{N}$ & - & - & $\mathrm{N}$ \\
\hline Cardillo et al. (1998) & $\mathrm{N}$ & $\mathrm{N}$ & $\mathrm{N}$ & - & - & $\mathrm{N}$ \\
\hline Zwaneveld et al. (2001) & $\mathrm{Y}$ & $\mathrm{N}$ & $\mathrm{N}$ & - & - & $\mathrm{N}$ \\
\hline Qiao (2008) & $\mathrm{Y}$ & $\mathrm{Y}$ & $\mathrm{N}$ & $\mathrm{N}$ & $\mathrm{N}$ & $\mathrm{N}$ \\
\hline Wu et al. (2013) & $\mathrm{N}$ & $\mathrm{N}$ & $\mathrm{N}$ & $\mathrm{N}$ & $\mathrm{N}$ & $\mathrm{N}$ \\
\hline Zhang (2018) & Y & $\mathrm{Y}$ & $\mathrm{Y}$ & $\mathrm{N}$ & Y & $\mathrm{N}$ \\
\hline This paper & $\mathrm{Y}$ & $\mathrm{Y}$ & $\mathrm{Y}$ & $\mathrm{Y}$ & $\mathrm{Y}$ & $\mathrm{Y}$ \\
\hline
\end{tabular}

${ }^{1}$ When column 'Arri./Dep. Route' contains a ' $Y$ ', it means that the routes in stations are divided into arrival route, departure routes. ${ }^{2}$ When column 'Occupation Time Analysis' contains ' $Y$ ', it means that the paper analyzes the occupation time of routes in detail. ${ }^{3}$ When column 'Pre-acclaim' contains ' $Y$ ', it means that the route occupation time consider the pre-acclaim time. ${ }^{4}$ When column 'Flexible Rules' contains ' $Y$ ', it means that flexible track utilization rule is adopted in the paper. ${ }^{5}$ When column 'Multi-direction' contains ' $Y$ ', it means that track allocation problem in multi-direction station is defined. ${ }^{6}$ When column 'Rolling Stock Plan' contains ' $Y$ ', it means that rolling stock plan is considered in the paper when solving track allocation problem.

A detailed explanation to the occupation time of different resources is given to facilitate the construction of the integer linear optimization model. The objective function is to minimize total trains' occupation time of resources in the throat area. The time constraints define the time window of resource-occupation by different trains and make sure the safety constraints can be easily coded by the commercial solver. The station layout constraint and static operation requirement are considered to ensure the feasibility of the results. A computational experiment with 49 trains in two hours is conducted to verify the effectiveness of the model. In a real case, the density of 49 trains in two hours is very high in a high-speed railway station. This train density can present the level of a large station. Beijing South Station, which is one of the biggest high-speed railway stations in China, has 25 trains in one hour, with 19 tracks for high-speed trains in total. The largest case in Wu et al. (2013) is 54 trains in 4 hours with 11 tracks [12]. In Leeds station, a busy station has 450 trains in a day (18.75 trains in one hour) with more than 12 platforms [9]. The branch-and-cut algorithm is used in the solver CPLEX to get the optimal solution. The SG' occupation plan and track allocation plan calculated by this model shows that all trains can be assigned with conflict-free resources within 1 second, which is much more efficient than experienced dispatchers within 10 minutes. The track allocation plans made by experienced dispatchers are quite error prone. The analysis of the solution denotes that the station capacity depends on the capacity of throat area rather than station tracks. Thus, it is significant for multi-direction stations to simultaneously optimize the usage of arrival-departure tracks and resources in the throat area. Our proposed method solves the track allocation problem in multi-direction station in a more practical way. Obviously, the constraints of train operations of high-speed railway stations in different countries are slightly different due to having different train control systems. Our proposed model can be slightly modified to satisfy the requirement of different stations.

On one hand, the existing limitation of this proposed model is that if the train timetable generated in the proceeding procedure is unfeasible, our proposed model cannot obtain a solution. However, under the current management method, the train timetable problem and track allocation problem are solved sequentially and iteratively. Thus, our proposed model can be used by timetable planners to generate a timetable with a feasible track allocation plan. On the other hand, our model does not differentiate whether the allocated train is high-speed or low-speed (such mainly used in freight). In a few high-speed railway stations, mixed traffic of high-speed and low-speed trains will be served. Thus, the operation constraints should be classified by introducing a parameter that indicates the train types. The objective function should also be reconstructed, such as in a multi-objective function, to satisfy different requirements of their services.

In the further research, we will focus on several aspects. First, a more complex model will be constructed to solve the track allocation problem of a multi-direction high-speed railway station 
and a customized solution method will be designed to solve the problem more efficiently. Second, the rescheduling track allocation problem will be investigated. The on-line problem of railway affects the service quality, especially for the high-speed railway. Third, the track allocation problem in multi-direction stations will be integrated into the train scheduling problem on the railway network to develop a better train scheduling plan.

Author Contributions: Conceptualization, Q.Z., X.Z. and L.W.; Data curation, Q.Z.; Formal analysis, Q.Z., X.Z. and L.W.; Funding acquisition, X.Z.; Investigation, Q.Z. and L.W.; Methodology, Q.Z., X.Z. and L.W.; Project administration, L.Wang; Resources, Q.Z.; Software, Q.Z.; Supervision, X.Z.; Validation, Q.Z. and X.Z.; Writing—original draft, Q.Z.; Writing—review \& editing, Q.Z.

Funding: This research was funded by the National Key R\&D Program of China, grant number 2018 YFB1201403.

Acknowledgments: I want to thank Rui Liu for technical support.

Conflicts of Interest: The authors declare no conflict of interest. The funders had no role in the design of the study; in the collection, analyses, or interpretation of data; in the writing of the manuscript, or in the decision to publish the results.

\section{References}

1. Woroniuk, C.; Aditjandra, P.T. A rail capacity framework: Making the case for rail freight evaluation in Indonesia. CSID J. Infrastruct. Dev. 2015, 1, 4-13. [CrossRef]

2. Marinov, M.; Şahin, İ.; Ricci, S.; Vasic-Franklin, G. Railway operations, time-tabling and control. Res. Transp. Econ. 2013, 41, 59-75. [CrossRef]

3. Meng, L.Y.; Zhou, X.S. Simultaneous train rerouting and rescheduling on an N-track network: A model reformulation with network-based cumulative flow variables. Transp. Res. Part B Methodol. 2014, 67, 208-234. [CrossRef]

4. Gao, Y.; Kroon, L.; Yang, L.X.; Gao, Z.Y. Three-stage optimization method for the problem of scheduling additional trains on a high-speed rail corridor. Omega 2017, 80, 175-191. [CrossRef]

5. Cardillo, D.D.L.; Mione, N. K L-list $\lambda$ colouring of graphs. Eur. J. Oper. Res. 1998, 106, 160-164. [CrossRef]

6. Billionnet, A. Using integer programming to solve the train-platforming problem. Transp. Sci. 2003, 37, 213-222. [CrossRef]

7. Zwaneveld, P.J.; Kroon, L.G.; Van Hoesel, S.P.M. Routing trains through a railway station based on a node packing model. Eur. J. Oper. Res. 2001, 128, 14-33. [CrossRef]

8. Lusby, R.; Larsen, J.; Ryan, D.; Ehrgott, M. Routing trains through railway junctions: A new set-packing approach. Transp. Sci. 2011, 45, 228-245. [CrossRef]

9. Carey, M.; Carville, S. Scheduling and platforming trains at busy complex stations. Transp. Res. Part A Policy Pract. 2003, 37, 195-224. [CrossRef]

10. Qiao, R. Research on Selection and Adjustment of Receiving Routes and Dispatching Routes in Passenger Dedicated Line Station. Ph.D. Thesis, Beijing Jiaotong University, Beijing, China, 2012. (In Chinese).

11. Liu, W. Optimizing the Bottleneck Sections and Track Allocation Problems for Large Railway Passenger Stations under Abnormal Conditions. Ph.D. Thesis, Beijing Jiaotong University, Beijing, China, 2017. (In Chinese)

12. Wu, J.J.; Kang, L.J; Sun, H.J.; Jia, X.L. Track allocation optimization in railway station: Mean-variance model and case study. J. Transp. Eng. 2013, 139, 540-547. [CrossRef]

13. Sels, P.; Vansteenwegen, P.; Dewilde, T.; Cattrysse, D.; Waquet, B.; Joubert, A. The train platforming problem: The infrastructure management company perspective. Transp. Res. Part B Methodol. 2014, 61, 55-72. [CrossRef]

14. Qi, J.G.; Yang, L.X.; Gao, Y.; Li, S.K.; Gao, Z.Y. Integrated multi-track station layout design and train scheduling models on railway corridors. Transp. Res. Part C Emerg. Technol. 2016, 69, 91-119. [CrossRef]

15. Zhang, Q.; Zhu, X.N.; Wang, L. Track allocation optimization in high-speed railway stations from infrastructure management and service perspectives. Meas. Control 2018, 51, 243-259. [CrossRef]

(C) 2019 by the authors. Licensee MDPI, Basel, Switzerland. This article is an open access article distributed under the terms and conditions of the Creative Commons Attribution (CC BY) license (http://creativecommons.org/licenses/by/4.0/). 